\title{
Thermosensibilité de l'expression ou de la transmission d'un facteur féminisant responsable de certaines formes de monogénie chez le Crustacé Oniscoïde Armadillidium vulgare Latreille.
}

\author{
P. JUCHAULT et J.J. LEGRAND \\ Laboratoire de Biologie Animale de l'Université de Poitiers et U.A. C.N.R.S. \\ $n^{\circ} 673$ "Physiologie et Génétique des Crustacés» \\ 40, avenue du Recteur Pineau, F 86022 Poitiers Cedex
}

\begin{abstract}
Résumé
Chez le Crustacé Isopode Oniscoïde Armadillidium vulgare, nous avons rapporté la monogénie à l'action de différents facteurs masculinisants et féminisants. Ces derniers transforment les mâles génétiques $(\mathrm{ZZ})$ en néo-femelles. L'un de ces facteurs (F) est une bactérie qui induit une thélygénie $\left(T_{F}\right)$ dont les néo-femelles sont réfractaires à la masculinisation. Les autres facteurs féminisants $\left(f^{\prime}-f\right)$ induisent respectivement une thélygénie $\left(T_{f^{\prime}}\right)$ et une arrhénogénie $\left({ }^{\prime} r_{f}\right)$ dont les néo-femelles sont masculinisables.
\end{abstract}

A $20{ }^{\circ} \mathrm{C}$, les femelles $T_{f}$, de la population de Rabat, expérimentées ici, ont fourni, pendant 4 générations, des descendances thélygènes où les mâles sont rarissimes et souvent absents. Lorsque ces femelles $T_{f}$, sont soumises à une température de $30^{\circ} \mathrm{C}$, leurs descendances $\left(F_{1}\right)$ deviennent, en $1^{\text {re }}$ ou $2^{\mathrm{e}}$ portée, arrhénogènes $\left(\mathrm{Ar}_{\mathrm{f}}\right)$. Lorsque la $1^{\mathrm{re}}$ portée est restée thélygène, les femelles qui en sont issues, élevées puis croisées à $20^{\circ} \mathrm{C}$, donnent des descendances $\left(F_{2}\right)$ du type $T_{f^{\prime}}$. Par contre, lorsqu'elles sont maintenues à $30^{\circ} \mathrm{C}$, elles donnent des $\mathrm{F}_{2}$ arrhénogènes $\left(\mathrm{AR}_{\mathrm{f}}\right)$. Lorsque la $1^{\text {re }}$ ou la $2^{\mathrm{e}}$ portée est devenue arrhénogène, les femelles « exceptionnelles » donnent en $\mathrm{F}_{2}$, à $20^{\circ} \mathrm{C}$, des descendances de sex-ratio variable: thélygène, arrhénogène ou intermédiaire (pseudo-amphogène) ; dans la plupart des cas, le sex-ratio des portées successives d'une même femelle reste constant. Le maintien, à $20^{\circ} \mathrm{C}$, de l'arrhénogénie s'apparente aux effets appelés "dauermodifications ». Cependant, dans certains cas, on observe à la $3^{\mathrm{e}}$ ou $4^{\mathrm{e}}$ portée une forte augmentation du taux des femelles, ce qui constitue un retour au type thélygène initial. Cette température de $30^{\circ} \mathrm{C}$ n'a aucun effet sur le sex-ratio des femelies génétiques $(W Z)$ qui reste amphogène $\left(\sigma^{\pi} / q=1\right)$.

Ces résultats montrent que c'est un seul et même facteur (f) qui est responsable des 2 formes de monogénie (thélygénie et arrhénogénie). L'expression de ce facteur est fonction de la température d'élevage ainsi que d'autres paramètres précédemment mis en évidence, tels que le génotype et l'âge de la génitrice. La multiplicité des paramètres en jeu conduit, dans certaines populations, à une variation continue du sex-ratio.

Cet ensemble de données peut être rapproché des résultats concernant la drosophile, à savoir la transmission du virus $\sigma$ et les systèmes P.M. et surtout I.R., responsables de différentes formes de dysgénésie hybride. La nature du facteur $\mathrm{f} \mathrm{d}^{\prime} A$. vulgare reste inconnue. Sa sensibilité à une haute température le différencie du déterminant sexuel des femelles génétiques.

Mots clés : Monogénie, température, facteur féminisant, crustacés, oniscoïdes. 


\section{Summary}

\section{Thermosensitivity of the expression (or the transmission) of a feminizing factor causing different types of monogeny \\ in the Crustacea Oniscoïdea Armadillidium vulgare Latreille.}

Monogeny observed in numerous populations of $A$. vulgare may be caused by various masculinizing $(\mathrm{M})$ and feminizing $\left(F, \mathrm{f}^{\prime}, \mathrm{f}\right)$ factors. The latter convert the genetic males $(\mathrm{ZZ})$ into neo-females. One of these factors (F) is a bacterium which induces a thelygeny where females are resistant to masculinization. The other feminizing factors $\left(f^{\prime}, f\right)$ respectively induce thelygeny $\left(T_{f^{\prime}}\right)$ and arrhenogeny $\left(\mathrm{Ar}_{\mathrm{f}}\right)$ with masculinizable females.

The $T_{f}$ females (population of Rabat) reared at $20^{\circ} \mathrm{C}$ during 4 generations produce thelygenous broods where males are exceedingly rare. When the $T_{f^{\prime}}$ females are reared at $30^{\circ} \mathrm{C}$, their progenies $\left(F_{1}\right)$ become arrhenogenous in the first or in the second brood. When the first brood remains thelygenous, the females reared and mated at $20^{\circ} \mathrm{C}$ produce $T_{f}$ broods $\left(F_{2}\right)$. Otherwise, when the females are reared at $30^{\circ} \mathrm{C}$, they produce $\mathrm{Ar}_{\mathrm{f}}$ broods $\left(\mathrm{F}_{2}\right)$.

When the first or the second brood has become arrhenogenous, the few females produce broods $\left(\mathrm{F}_{2}\right)$ with variable sex-ratio : thelygenous, arrhenogenous or intermediate (pseudo-amphogenous) ; in most cases, the sex-ratio of the successive broods of a female remains unchanged. The persistence of arrhenogeny, at $20^{\circ} \mathrm{C}$, shows similarities with the effects named «dauermodifications".

However in some cases, the sex-ratio of the 3rd or 4th brood becomes increasingly female biased. Such variations constitute a reversion to the initial thelygenous type. This temperature $\left(30^{\circ} \mathrm{C}\right)$ has no effect on the sex-ratio of the genetic females $(\mathrm{ZW})$ which remains amphogenous $\left(0^{\prime \prime} /\right.$ O = 1).

These results show that one factor (f) only is the cause of the 2 types of monogeny (thelygeny and arrhenogeny). The expression of this factor depends on temperature and other parameters such as genotype and age of females. The multiplicity of the acting parameters causes in some populations a continued variation of sex-ratio.

These data seem to have similarities with those related to Drosophila, namely transmission of virus $\sigma$ and the P.M. and above all I.R. systems, which determine hybrid dysgenesis. The nature of the $\mathrm{f} A$. vulgare factor is unknown. Its sensitivity to high temperature distinguishes it from the sexual determinant of the genetic females.

Key words : Monogeny, temperature, feminizing factor, Crustacea, Oniscoïdea.

\section{Introduction}

La monogénie, phénomène très répandu chez les Crustacés Péracarides - en particulier chez les Isopodes Oniscoïdes - consiste en la production, par un couple, de descendances composées en totalité, ou en grande majorité, par des individus de même sexe. Ces descendances sont qualifiées de thélygènes $(T)$ lorsqu'elles sont constituées de femelles, et d'arrhénogènes (Ar) lorsqu'il s'agit de mâles. Dans une descendance dite allélogène, les portées successives d'une même femelle diffèrent significativement quant au sex-ratio.

VANDEL (1941) oppose à ces divers types de descendances monogènes les descendances amphogènes qui offrent une égalité numérique des sexes. Diverses interprétations faisant appel à un déterminisme à la fois génétique polyfactoriel et épigénétique ont été proposées pour expliquer ces phénomènes de monogénie (LegRAND \& Juchault, 1984 ; Ginsburger-Vogel, 1985 ; Bull, 1983). Chez l'Oniscoïde Armadillidium vulgare, où l'amphogénie est rare, limitée à certaines lignées présentes dans quelques populations, et caractérisée par l'existence de femelles et de mâles génétiques 
( $q \mathrm{WZ}, \sigma^{\prime} \mathrm{ZZ}$ ), nous avons rapporté la monogénie à l'action de différents facteurs féminisants et masculinisants. Les facteurs masculinisants, à transmission patrocline, s'opposent dans certains croisements à l'action des facteurs féminisants, induisant alors l'intersexualité et parfois un maintien du phénotype mâle ; ils sont de nature génétique et correspondent à un gène dominant $M$ (Juchault \& Legrand, 1976b ; Legrand \& JuCHAULT, 1984). Les facteurs féminisants, à transmission essentiellement matrocline, transforment les mâles génétiques en néo-femelles. L'un de ces facteurs féminisants est une bactérie Chlamydiale (F), intracytoplasmique et polytrope, responsable des descendances thélygènes $\left(\mathrm{T}_{\mathrm{F}}\right)$, dont les femelles ne peuvent être masculinisées (MARTIN et al., 1973 ; MARTIN, 1981). Cette forme de monogénie peut être induite par implantation de tissu de femelle $T_{F}$ chez une femelle amphogène (Legrand \& Juchault, 1970). Les autres facteurs féminisants (f et $\mathrm{f}^{\prime}$ ) transforment les mâles génétiques en néo-femelles masculinisables, induisant respectivement l'arrhénogénie $\mathrm{Ar}_{\mathrm{f}}$ et la thélygénie $\mathbf{T}_{\mathrm{f}^{\prime}}$. Ces 2 dernières formes de monogénie ne peuvent pas être induites chez un receveur amphogène par implantation de tissus de néo-femelles $\operatorname{Ar}_{\mathrm{f}}$ ou $\mathrm{T}_{\mathrm{f}^{\prime}}$.

Le facteur $\mathbf{f}^{\prime}$ est stable : étant régulièrement transmis par les néo- $\mathbf{Z Z}_{\mathrm{f}^{\prime}}$ il provoque une thélygénie $\left(\mathrm{T}_{\mathrm{f}^{\prime}}\right)$. Les croisements entre individus issus de populations différentes ont montré que la transmission de $\mathbf{f}^{\prime}$ est contrôlée par un mécanisme génétique (Juchault \& Legrand, 1976a). Une certaine proportion (1/3) des mâles issus de descendances $T_{f^{\prime}}$ peuvent transmettre ce type de thélygénie (Juchault \& Legrand, 1981b ; Legrand \& JuChault, 1984) ; mais la plupart de ces mâles d'origine $\mathrm{T}_{\mathrm{f}}$ ' sont « neutres » : croisés avec des femelles génétiques, ils donnent des descendances amphogènes (Legrand \& JuchaulT, 1972).

Le facteur $f$ peut être qualifié de labile. Il semble absent - ou non exprimé dans de nombreux ovocytes des femelles $\mathrm{Ar}_{\mathrm{f}}$ de telle sorte que le croisement : $\sigma^{7}$ $\mathrm{ZZ} \times$ néo- $O \mathrm{ZZ}_{\mathrm{f}}$ donne des descendances arrhénogènes $\left(\mathrm{Ar}_{\mathrm{f}}\right)$ du fait que les zygotes, $\mathrm{ZZ}$, ne peuvent se développer qu'en accord avec leur sexe génétique (mâle). Les mâles arrhénogènes $\mathrm{Ar}_{\mathrm{f}}$ - comme la plupart des mâles d'origine $\mathrm{T}_{\mathrm{f}^{\prime}}-$ sont neutres. Leur taux dans ces descendances $\mathrm{Ar}_{\mathrm{f}}$ augmente souvent au cours des portées successives ; généralement, les dernières portées des femelles $\operatorname{Ar}_{\mathrm{f}}$ âgées ne sont constituées que par des mâles. Ce phénomène, désigné par VANDEL (1941) sous le nom d'arrhénauxèse, rend l'ensemble de la descendance allélogène.

Nous avons observé dans certaines filiations un passage spontané de la thélygénie $\mathrm{T}_{\mathrm{f}}$ '̀̀ l'arrhénogénie $\mathrm{Ar}_{\mathrm{f}}$ et, plus rarement, un changement inverse (LeGRAND \& Juchault, 1984). L'ensemble de ces données concernant les facteurs $f-f^{\prime}$ rappellent les résultats relatifs à la transmission du virus $\sigma$ de la drosophile (L'Héritier, 1962 ; Ohanessian-Guillemain, 1963). Cette similitude sera développée dans la discussion. La transmission du virus étant profondément modifiée par un traitement thermique $\left(30^{\circ} \mathrm{C}\right)$, nous avons recherché s'il pouvait en être de même en ce qui concerne le facteur féminisant $\mathrm{f}^{\prime}$.

Rappelons que de telles expériences ont déjà été réalisées par nous-mëmes (Juchault et al., 1980a), chez des femelles d'A. vulgare de la lignée thélygène $T_{\mathrm{F}}$ et par Ginsburger-Vogel (1975) et Ginsburger-Vogel \& Magniette-Mergault (1981a 1981b) chez les femelles thélygènes de l'amphipode Orchestia gammarellus. Mais dans ces deux cas, les facteurs féminisants - qui inversent les mâles génétiques en néo- $q$ correspondaient à des symbiotes parfaitement identifiés : microsporidie chez $O$. gammarellus, bactérie chez $A$. vulgare. Chez les néo-femelles de cette dernière espèce, une température de $35^{\circ} \mathrm{C}$ appliquée pendant 35 jours provoque la disparition des « formes 
typiques » de la bactérie. Cette disparition est concomitante d'une différenciation de caractères mâles, conformes au génotype. Cependant, après retour à $20^{\circ} \mathrm{C}$, de nouvelles formes typiques de la bactérie réapparaissent et la physiologie femelle se rétablit.

\section{Matériel et méthodes}

Les $A$. vulgare utilisés dans cette étude sont des descendants d'individus récoltés à Rabat (Maroc). Les croisements effectués durant 3 ans à partir de l'échantillon recueilli n'ont fourni que des descendances monogènes à femelles masculinisables, les unes thélygènes, les autres arrhénogènes. Aucune souche amphogène stable, comparable à celles obtenues à partir des femelles génétiques (WZ) d'autres populations, n'a pu être isolée à partir de cette population marocaine. Compte tenu des résultats antérieurs concernant les populations d'A. vulgare, on peut penser que tous les individus de la population de Rabat sont des mâles génétiques et que le phénotype femelle résulte uniquement de l'action de facteurs féminisants de type $f-f^{\prime}$, puisque la bactérie $F$ semble absente. Nous avons déjà observé de telles populations, en particulier à Moulis (09200 France) et à Escorial (Espagne) (Juchault \& Legrand, 1981b).

Lors des croisements préalables, à $20^{\circ} \mathrm{C}$, des femelles issues d'une descendance $\mathrm{T}$ avec des mâles issus de descendances $\mathrm{Ar}$ de la même population, la thélygénie s'est montrée parfaitement stable : $F 1=73 q ; F 2$ obtenues à partir de 5 femelles $F_{1}=7 \sigma^{\prime}$, $358 \%$; F3 obtenues à partir de 10 femelles $F_{2}=8 \sigma^{\prime \prime}, 1698$. L'expérience a consisté à soumettre à une température de $30^{\circ} \mathrm{C}$ des femelles vierges pubères, en repos sexuel, issues de descendances $T_{f^{\prime}}$, en les associant chacune avec un mâle de la même population (mâles issus de descendances Ar). Ce type de mâle a été choisi du fait qu'il est «neutre " et pour éviter l'introduction d'un génotype étranger, susceptible de modifier la transmission de $\mathrm{f}^{\prime}$ (JuchaulT \& LEGRAND, 1976a). Les premières portées sont intervenues 45 jours après la mise en expérience. Les descendants des femelles $T_{f^{\prime}}$ ont été maintenus à $30^{\circ} \mathrm{C}$ pendant les 30 à 40 premiers jours de leur vie ; les mâles et les femelles pouvant alors être distingués, les descendants ont été séparés par sexe et, soit ramenés à $20^{\circ} \mathrm{C}$ où ils ont effectué leur croissance, soit maintenus à $30^{\circ} \mathrm{C}$. Des croisements ultérieurs ont été réalisés avec ces individus, soit à $20^{\circ} \mathrm{C}$, soit à $30^{\circ} \mathrm{C}$. Nous avons également utilisé, dans certains tests, des femelles d'une souche amphogène vraie, issue d'une population espagnole (Valence) (Juchault \& LEGRAND, 1981b). Afin d'obtenir une entrée rapide en reproduction et un allongement de cette période de reproduction, tous les élevages ont été effectués sous une photopériode de type jours longs (L.D. : 18.6) (MocQuARD et al., 1980). Nous avons préalablement observé que de telles photopériodes n'ont aucun effet sur le sex-ratio des descendances amphogènes ou monogènes (résultats inédits).

\section{Résultats}

\section{A. Descendances des femelles $T_{f^{\prime}}$ élevées à $20^{\circ} \mathrm{C}$ (témoins)}

Vingt femelles issues d'une $\mathrm{F}_{3}$ thélygène, élevées à $20^{\circ} \mathrm{C}$ et croisées avec un mâle de lignée $\mathrm{Ar}_{\mathrm{f}}$ ont donné chacune une descendance $\mathrm{T}$; l'ensemble des descendants, qui constitue la $4^{\mathrm{e}}$ génération $T_{f^{\prime}}$ obtenue au laboratoire, représente $6 \sigma^{7}$ et $1067 q$ pour la 
$1^{\text {re }}$ portée des 20 femelles étudiées, $468 q$ pour la $2^{\mathrm{e}}$ portée chez 8 des femelles précédentes et $105 q$ pour la $3^{\mathrm{e}}$ portée chez 4 d'entre elles.

\section{B. Descendances des femelles $T_{f^{\prime}}$ pubères transférées à $30^{\circ} \mathrm{C}$ \\ $\left(F_{1}\right.$ des femelles soumises à $30^{\circ} \mathrm{C}$ )}

Vingt-deux femelles $T_{f^{\prime}}$, sœurs des précédentes, ont été transférées à $30^{\circ} \mathrm{C}$ et croisées également chacune avec un mâle de lignée $\mathrm{Ar}_{\mathrm{f}}$. Elles ont donné des descendances dont le sex-ratio de la $1^{\text {re }}$ portée est variable, mais souvent constitué d'un nombre important de mâles ; le pourcentage moyen de mâles est de 33,2 p. 100 (voir tableau 1). Chez 11 femelles qui ont offert une $2^{\mathrm{e}}$ portée, on observe une augmentation significative du taux des mâles lors de cette $2^{\mathrm{e}}$ portée (taux moyen : 84,8 p. 100). Cette arrhénauxèse concerne toutes les descendances, quelle que soit le sex-ratio de la $1^{\text {re }}$ portée ; elle aboutit souvent à une arrhénogénie totale (exemple : $1^{\text {re }}$ portée $55 q ; 2^{\mathrm{e}}$ portée $\left.53 \sigma^{7}\right)$.

Les descendances amphogènes observées dans certaines premières portées n'apparaissent donc que comme des états de transition du sex-ratio entre la thélygénie et l'arrhénogénie. On peut ainsi parler de pseudoamphogénie.

\section{Tableau 1}

Action d'une température de $20^{\circ} \mathrm{C}$ et $30^{\circ} \mathrm{C}$ sur le sex-ratio des descendances de femelles génétiques (WZ) et de femelles (néo-femelles $Z Z$ ) de lignée $T_{f}$. $n$ : nombre de descendances; $T$, pseudo $A, A r$ : descendances dont le sex-ratio est respectivement thélygène, pseudo-amphogène, arrhénogène $;+$ la $1^{\text {re }}$ portée de cette descendance avait été thélygène; ++ les $1^{\text {res }}$ portées avaient été thélygènes (8 cas), amphogène (1 cas), arrhénogène (1 cas).

Effect of temperature $\left(20^{\circ} \mathrm{C}\right.$ and $\left.30^{\circ} \mathrm{C}\right)$ on the sex-ratio of progenies of genetic females (WZ) and $T_{f}$ females (neo-females $Z Z$ ). $n$ : number of progenies ; $T$, pseudo $A, A r$ :

progenies with sex-ratio respectively thelygenous, pseudo-amphogenous, arrhenogenous ;

+ the first brood of the progeny was thelygenous; ++ the first broods were thelygenous ( 8 cases), amphogenous (one case), arrhenogenous (one case).

\begin{tabular}{|c|c|c|c|c|c|c|}
\hline \multirow[b]{2}{*}{$\begin{array}{r}20^{\circ} \mathrm{C} 1^{\text {re }} \\
2^{e} \\
3^{e}\end{array}$} & \multirow[b]{2}{*}{$\begin{array}{l}\text { portée } \ldots \ldots \ldots \\
\text { portée } \ldots \ldots \ldots \\
\text { portée } \ldots \ldots \ldots\end{array}$} & \multicolumn{2}{|c|}{$\begin{array}{c}\text { Descendances } \\
\text { des } \$ \text { génétiques }\end{array}$} & \multicolumn{3}{|c|}{$\begin{array}{c}\text { Descendances } \\
\text { des } Q \text { (néo-Q) } \mathrm{T}_{\mathrm{f}^{\prime}}\end{array}$} \\
\hline & & $\begin{array}{l}\mathrm{n}=12 \\
\mathrm{n}=10 \\
=9\end{array}$ & $\begin{array}{llll}355 & \sigma^{7} & 362 & q \\
273 & \sigma^{\prime} & 268 & + \\
264 & \sigma^{\prime} & 271 & q\end{array}$ & $\begin{array}{l}\mathbf{n}=20 \\
\mathbf{n}=8 \\
\mathbf{n}=4\end{array}$ & 60 & $\begin{array}{r}1067 \stackrel{+}{9} \\
486 \stackrel{+}{+} \\
105 \text { 안 }\end{array}$ \\
\hline $30^{\circ} \mathrm{C} 1^{\mathrm{re}}$ & portée & $\mathrm{n}=17$ & 522 ơ 505 우 & $\begin{array}{l}\mathrm{n}=22 \\
14 \mathrm{~T} \\
4 \mathrm{pseudo} \mathrm{A} \\
4 \mathrm{Ar}\end{array}$ & $\begin{aligned} 371 & \sigma^{7} \\
97 & \sigma^{\prime \prime} \\
129 & \sigma^{\prime \prime} \\
145 & \sigma^{\prime \prime}\end{aligned}$ & $\begin{array}{rl}747 & q \\
607 & q \\
116 & q \\
24 & q\end{array}$ \\
\hline & portée & $\mathrm{n}=8$ & $179 \sigma^{\prime} 173$ 웅 & $\begin{array}{l}\mathrm{n}=11 \\
1 \text { pseudo } \mathrm{A} \\
10 \mathrm{Ar}\end{array}$ & $\begin{aligned} 246 & \sigma^{7} \\
20 & \sigma^{\prime \prime} \\
226 & \sigma^{\prime \prime}\end{aligned}$ & $\begin{array}{l}46 q \\
19 \stackrel{q}{q}+ \\
27 q++\end{array}$ \\
\hline $3^{e}$ & portée ...... & $\mathrm{n}=6$ & $190 \sigma^{\prime} 196 q$ & & & \\
\hline
\end{tabular}




\section{Descendances de femelles génétiques (WZ) transférées à $30^{\circ} \mathrm{C}$}

Afin de vérifier si l'action d'une température élevée pouvait avoir un effet direct sur le sex-ratio des descendances de femelles génétiques ( $Q$ WZ), nous avons élevé à $30^{\circ} \mathrm{C}$, à titre de comparaison, 17 femelles de ce type, issues de la souche amphogène stable de Valence (Espagne). Croisées à $30^{\circ} \mathrm{C}$ avec des mâles de la lignée arrhénogène de Rabat, elles ont toutes donné, comme à $20^{\circ} \mathrm{C}$, des descendances amphogènes au cours de leurs trois portées successives (voir tableau 1).

\section{Descendances des mâles issus des femelles $T_{f}$, soumises à $30^{\circ} \mathrm{C}(F 2)$}

14 mâles nés à $30^{\circ} \mathrm{C}$ dans 4 descendances arrhénogènes ( $1^{\mathrm{re}}$ ou $2^{\mathrm{e}}$ portée) obtenues au paragraphe $\mathrm{B}$, ont été croisés, à $20^{\circ} \mathrm{C}$, avec des femelles génétiques (souche amphogène de Valence). Les 14 descendances ont été amphogènes et l'amphogénie s'est maintenue au cours des 3 ou 4 portées successives; l'ensemble des 14 premières portées représente $372 \sigma^{7}$ et 392 \&.

\section{E. $F_{2}$ des femelles soumises au choc thermique de $30^{\circ} \mathrm{C}$}

\section{Cas des femelles provenant d'une descendance restée thélygène en $I^{\text {re }}$ portée}

- 8 femelles nées à $30^{\circ} \mathrm{C}$ et appartenant à une descendance $\mathrm{T}$ pure $\left(1^{\text {re }}\right.$ portée $=55$ Q), ayant accompli leur croissance à $20^{\circ} \mathrm{C}$, ont été croisées à cette tempéra-

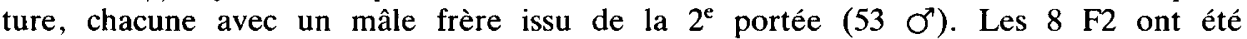
thélygènes (ensemble des $1^{\text {res }}$ portées $=209$ q) et cette thélygénie pure s'est maintenue au cours des 3 à 4 portées suivantes.

- 5 femelles, sœurs des précédentes mais restées à $30^{\circ} \mathrm{C}$, ont également été croisées à cette température avec un mâle frère. A l'inverse de ce qui a été observé à $20^{\circ} \mathrm{C}$, les $5 \mathrm{~F}_{2}$ ont été arrhénogènes ( $1^{\text {res }}$ portées $=103 \sigma^{\prime}$ et 18 O ).

\section{Cas des femelles provenant d'une descendance devenue arrhénogène (tabl. 2)}

30 femelles nées à $30^{\circ} \mathrm{C}$ dans 4 des descendances $\operatorname{Ar}\left(1^{\text {re }}\right.$ ou $2^{\mathrm{e}}$ portée), ayant accompli leur croissance à $20^{\circ} \mathrm{C}$, ont été croisées, à cette température, avec un mâle frère. Les $30 \mathrm{~F}_{2}$ ont donné des descendances dont le sex-ratio de la $1^{\text {re }}$ portée est très variable : thélygène ( $6 \mathrm{cas})$, pseudoamphogène $(8 \mathrm{cas})$, arrhénogène (16 cas) (voir tableau 2). 21 génitrices ont pu être maintenues en élevage pendant 1 an et ont donné 4 à 6 portées. Dans la plupart des cas, le sex-ratio de ces portées n'a pas évolué.

Cependant, sur 8 génitrices ayant donné une $1^{\text {re }}$ portée arrhénogène, nous avons constaté dans la descendance de 4 d'entre elles, une forte augmentation du taux des femelles à partir de la $3^{\mathrm{e}}$ ou $4^{\mathrm{e}}$ portée. Cette allélogénie aboutit, dans un cas, à de l'amphogénie, et dans 3 cas, à une forte thélygénie, comme le montre l'exemple suivant concernant les portées successives d'une de ces femelles: $72 \sigma^{\prime \prime}, 5 q ; 15 \sigma^{\prime \prime}, 9 q ; 3$

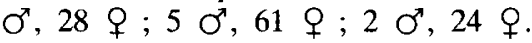

Des croisements complémentaires ont montré que l'amphogénie apparue dans certaines de ces $F_{2}$, bien que stable au cours des portées successives, ne correspond pas à une vraie amphogénie résultant du croisement entre un mâle et une femelle généti- 
TABleaU 2

Descendances à $20^{\circ} \mathrm{C}$ ( $I^{r e}$ portée) des femelles nées à $30^{\circ} \mathrm{C}\left(F_{2}\right)$ et dont la mère était issue d'une descendance $T_{f^{\prime}}$ (cas des descendances $F_{2}$ arrhénogènes) $\left(\$ E_{2}\right)$. (même légende que le tableau 1).

Progenies at $20^{\circ} \mathrm{C}$ (first brood) of females born at $30^{\circ} \mathrm{C}\left(F_{2}\right)$, the mother of which originates from a $T_{f^{\prime}}$ progeny $\left(F_{2}\right.$ arrhenogenous progenies; $\left.\S E_{2}\right)$ (same legend as in table 1$)$.

\begin{tabular}{|c|c|c|c|c|}
\hline \multirow{2}{*}{$\begin{array}{c}\begin{array}{c}\text { Descendances } \mathrm{F}_{1} \text { de } \\
\text { élevées à } 30^{\circ} \mathrm{C}\end{array} \mathrm{T}_{\mathrm{f}^{\prime}} \\
\begin{array}{c}31 \sigma^{\prime} 16 \% \\
\left(2^{\mathrm{e}} \text { portée }\right)\end{array}\end{array}$} & \multicolumn{4}{|c|}{$\begin{array}{l}\text { Descendances } \mathrm{F}_{2}\left(1^{\mathrm{re}} \text { portée }\right) \\
\text { à } 20^{\circ} \mathrm{C} \text {, de } Q^{\circ} \text { nées à } 30^{\circ} \mathrm{C}\left(\mathrm{F}_{1}\right)\end{array}$} \\
\hline & $n=13$ & $\begin{array}{l}6 \text { pseudo A } \\
6 \mathrm{Ar} \\
1 \mathrm{~T}\end{array}$ & $\begin{aligned} 225 & \sigma^{\prime \prime} \\
192 & \sigma^{\prime \prime} \\
2 & \sigma^{\prime \prime}\end{aligned}$ & $\begin{array}{rl}195 & q \\
85 & q \\
80\end{array}$ \\
\hline $\begin{array}{l}51 \sigma^{\circ} 11 \%+ \\
\left(1^{\mathrm{re}} \text { portée }\right)\end{array}$ & $n=4$ & $\begin{array}{ll}1 \mathrm{~A} \\
3 \mathrm{~T}\end{array}$ & $\begin{array}{l}21 \sigma^{\prime \prime} \\
100^{\prime \prime}\end{array}$ & $\begin{aligned} & 18 \circ \\
& 137 \stackrel{+}{q}\end{aligned}$ \\
\hline $\begin{array}{c}25 \sigma^{7} 13 \text { 早 } \\
\left(1^{\text {re }} \text { portée }\right)\end{array}$ & $\mathrm{n}=11$ & $\begin{array}{l}10 \mathrm{Ar} \\
1 \text { pseudo } \mathrm{A}\end{array}$ & $\begin{aligned} 418 & \sigma^{\prime \prime} \\
18 & \sigma^{\prime \prime}\end{aligned}$ & $\begin{array}{ll}29 & q \\
24 & + \\
q\end{array}$ \\
\hline $\begin{array}{l}210^{\prime} 3 q \\
\left(2^{\mathrm{c}} \text { portée) }\right.\end{array}$ & $\mathrm{n}=2$ & $2 \mathrm{~T}$ & $38 \sigma^{7}$ & 68 우 \\
\hline
\end{tabular}

que. En effet, les femelles issues de ces $F_{2}$ amphogènes ont produit des descendances Ar lorsqu'elles ont été croisées à $30^{\circ} \mathrm{C}$ avec leurs frères : 10 croisements ont donné 10 descendances Ar représentant $252 \sigma^{\prime \prime}$ et 65 Q

Enfin, les $\mathrm{F}_{3}$ effectuées à partir des femelles nées à $20^{\circ} \mathrm{C}$ dans des descendances Ar puis transférées après la puberté à $30^{\circ} \mathrm{C}$ ont également donné des descendances $\mathrm{Ar}$ avec une accentuation du taux des mâles en $1^{\text {re }}$ portée $\left(295 \sigma^{*}, 11\right.$ q) et même une disparition des femelles en $2^{e}$ portée $\left(146 \sigma^{n}\right)$.

\section{Discussion}

Ces résultats montrent qu'une température élevée $\left(30^{\circ} \mathrm{C}\right)$ modifie le sex-ratio des descendances des femelles de la lignée thélygène à facteur $\mathbf{f}^{\prime}$ (néo-femelle $Z_{\mathrm{f}^{\prime}}$ ). Cette même température n'a aucun effet sur la descendance des femelles génétiques (WZ), c'est-à-dire qu'elle ne modifie pas l'expression du déterminant femelle porté par l'hétérochromosome $\mathrm{W}$. La température de $30^{\circ} \mathrm{C}$ peut donc inhiber, soit la transmission soit l'expression du facteur féminisant $\mathrm{f}^{\prime}$ chez les descendants, de telle sorte que ces derniers acquièrent un phénotype mâle conforme à leur génotype (ZZ). Ainsi, l'étude de l'action de la température sur les femelles $T_{f^{\prime}}$ confirme que ces dernières sont bien des néo-femelles. Les modalités de la disparition - ou de l'absence d'expression - de $\mathbf{f}^{\prime}$ devront être étudiées ultérieurement. Ce facteur ne semble pas avoir été transmis à leurs fils par les femelles soumises à $30^{\circ} \mathrm{C}$ puisque ces mâles ont toujours donné, à $20^{\circ} \mathrm{C}$, des descendances amphogènes $(\S$ III D). Ce résultat suggère que le passage de la thélygénie $T_{f^{\prime}}$ à l'arrhénogénie $A r_{f}$ ne correspond pas à l'effet de la température sur un gène thermosensible. 
L'action de la température élevée s'exerce plus ou moins rapidement; elle est souvent nette dès la $1^{\text {re }}$ portée et, dans tous les cas, intervient lors de la $2^{\mathrm{e}}$ portée qui, fréquemment, ne comporte que des mâles. Sous l'effet de la température de $30^{\circ} \mathrm{C}$, il y a donc passage de la thélygénie $T_{f}$ ' à l'arrhénogénie $A r_{f}$, c'est-à-dire passage d'une forme stabilisée $\left(f^{\prime}\right)$ d'un facteur féminisant à une forme non stabilisée (f).

L'arrhénauxèse ainsi observée rappelle l'augmentation du nombre de mâles rencontrée dans les dernières portées des femelles âgées des lignées arrhénogènes (LEGRAND \& JuCHAULT, 1969).

Lorsque la température n'a pas eu d'effet sur la $1^{\text {re }}$ portée de la $F_{1}$ (cas des portées restées thélygènes), le croisement à $20^{\circ} \mathrm{C}$ des néo-femelles $\mathrm{F}_{1}$ avec des mâles frères de la $2^{\mathfrak{e}}$ portée montre qu'il y a maintien en $F_{2}$ de la forme stabilisée du facteur féminisant. Cependant, ces néo-femelles $F_{1}$ ne peuvent pas être considérées comme réfractaires à l'action de la température de $30^{\circ} \mathrm{C}$ puisqu'elles fournissent des $F_{2}$ arrhénogènes lorsqu'elles sont maintenues et croisées à cette température. La précocité - ou l'effet retardé - de la température sur le phénotype sexuel des descendants peut dépendre du génotype ou de l'état physiologique de l'animal qui varie en fonction des différentes phases de l'ovogenèse. Cependant, l'effet de la température finit toujours par intervenir - ou par s'accentuer - lors de la $2^{\mathrm{e}}$ portée. Ceci suggère, soit qu'en présence de certains génotypes le choc thermique doit avoir une durée suffisamment longue pour être totalement efficace, soit que la période de sensibilité du facteur $\mathrm{f}^{\prime}$ à la température se situe à certaines phases du cycle ovogénétique. Le fait qu'il faut attendre, dans certains cas, le $2^{\mathrm{c}}$ cycle ovogénétique pour observer un effet de la température suggère que la phase sensible pourrait se placer au tout début du cycle.

Lorsque la température a un effet inhibiteur partiel (variable d'un zygote à l'autre) sur le facteur $\mathrm{f}^{\prime}$ (cas des portées Ar imparfaites), il est difficile de prévoir la nature des descendances ultérieures des néo-femelles « exceptionnelles ». En effet, lorsqu'elles sont croisées à $20^{\circ} \mathrm{C}$, on observe, dans les premières portées, une grande hétérogénéité dans la transmission - ou l'expression - des facteurs féminisants puisque les descendances peuvent être thélygènes, amphogènes ou arrhénogènes. Ce résultat semble donc indiquer que ces génitrices - dont la mère possédait des facteurs du type stabilisé ( $\left.f^{\prime}\right)$ peuvent, selon les cas, avoir hérité de facteurs de type stabilisé ou non stabilisé (f). Cependant l'étude, sur un an, des descendances de ces femelles montre qu'il peut intervenir chez certaines une modification du sex-ratio après la $3^{e}$ ou la $4^{e}$ portée ; cette évolution correspond toujours à une forte augmentation du taux des femelles, c'est-àdire à un retour tardif au type stabilisé $\mathrm{f}^{\prime}$. Le maintien d'une arrhénogénie stable dans les descendances des néo-femelles nées à $30^{\circ} \mathrm{C}$ puis élevées pendant un an à $20^{\circ} \mathrm{C}$ peut être considéré comme un effet retardé de la température, mais susceptible d'être réversible chez certaines femelles. Cet effet retardé n'est pas sans rappeler les effets appelés « dauermodifications » et rapportés parfois à des éléments extrachromosomiques (BREgliano et al., 1980 ; Minamori, 1969).

L'ensemble des résultats concernant l'action d'une température de $30^{\circ} \mathrm{C}$ sur les facteurs féminisants $f-f^{\prime}$ conduit donc à penser qu'il n'y a pas 2 facteurs distincts mais un seul et même facteur que nous appellerons désormais $\mathrm{f}$. La transmission - ou l'expression - de ce facteur dépendrait de divers paramètres tels que l'âge de la femelle, le génotype de l'hôte, la température d'élevage. Les modalités d'action de ces différents paramètres conditionnent ainsi la forme de la monogénie : arrhénogénie ou thélygénie. Celle-ci est fonction de l'activité - ou de l'inhibition, ou encore de l'absence - du facteur $f$ dans un nombre variable d'ovocytes. Il est à souligner qu'étant 
donné la multiplicité des paramètres en jeu, ces 2 formes de monogénie ne représentent - au moins dans certaines populations (Juchault \& Legrand, 1981b) - que les 2 extrêmes d'une variation quantitative continue de l'expression du facteur féminisant. L'existence de descendances pseudo-amphogènes est une manifestation de cette variation continue. Une transmission patrocline - et généralement atténuée - de ce facteur f a également été observée chez certains mâles de la lignée thélygène $T_{f^{\prime}}$, ainsi que chez des néo-mâles expérimentaux. Une telle transmission n'a pas été observée chez les mâles fils des femelles $T_{f^{\prime}}$, soumises à $30^{\circ} \mathrm{C}$ (paragraphe III D). Ceci rejoint les données précédentes concernant l'état «neutre " des mâles issus des descendances $\mathrm{Ar}_{\mathrm{f}}$ : croisés avec des femelles génétiques, ils donnent toujours de l'amphogénie (LEGRAND \& JuchaUlT, 1972).

Les résultats concernant les modalités de transmission de ces facteurs féminisants ne sont pas sans rappeler certains faits se rapportant au virus $\sigma$ responsable de la sensibilité au $\mathrm{CO}^{2}$ chez la Drosophile (L'Héritier, 1962 ; Ohanessian-Guillemain, 1963). Nous avons déjà développé (Legrand \& Juchault, 1984) les similitudes existant entre la transmission des facteurs $f$ et $f^{\prime}$ et la transmission du virus $\sigma$ de la drosophile. Rappelons que la stabilité de la transmission par la néo-femelle du facteur $\mathrm{f}^{\prime}$ a son équivalent dans la transmission maternelle de l'état stabilisé du virus $\sigma$. De plus, les modalités de la transmission maternelle de plus en plus faible du facteur $\mathrm{f}^{\prime}$ aboutissant à l'arrhénogénie $\mathrm{Ar}_{\mathrm{f}}$ rappelle les modalités de la transmission maternelle de l'état non stabilisé du virus. De même l'absence de transmission de cet état par les mâles est comparable à l'état "neutre " des mâles $\mathrm{Ar}_{\mathrm{f}}$. Les résultats, relatés ici, concernant l'effet d'une température de $30^{\circ} \mathrm{C}$ rejoignent également ceux relatifs au virus $\sigma$.

Ils peuvent être également rapprochés de certaines données concernant la dysgénésie hybride de Drosophila melanogaster induite par des systèmes faisant appel à des éléments mobiles d'ADN : d'une part, le système P.M., où la dysgénésie (stérilité gonadique, due à l'atrophie de l'ovaire) résulte de l'interaction d'éléments mobiles $(\mathrm{P})$, associés aux chromosomes des mâles, et d'un état maternel extrachromosomique sensible (cytotype M) (Anxolabehere \& PériQuet, 1983) ; d'autre part, le système I.R., où la dysgénésie (mortalité embryonnaire) est produite par l'interaction entre des éléments transposables inducteurs (I) et un état cellulaire réacteur (R) (BREgLiano et al., 1980). Dans ce système I.R., l'état cytoplasmique des femelles évolue en fonction de leur âge et est sensible aux traitements thermiques : plus la femelle est âgée, plus la "réactivité " décroît ; de même, une température de $30^{\circ} \mathrm{C}$ appliquée durant la fin de l'ovogenèse entraîne une diminution de cette « réactivité ». Ces effets sont partiellement héréditaires et toujours réversibles. Toutes ces modalités caractérisant l'état réactif $(R)$ des femelles dans le système I.R. rappellent donc d'une façon particulièrement nette les modalités d'action du facteur f. La nature des déterminants de cette "réactivité » des drosophiles est encore inconnue. Deux hypothèses ont été proposées : la première fait appel à des déterminants chromosomiques, la seconde à des éléments génétiques extrachromosomiques, symbiotes ou organelles (Bregliano et al., 1980).

Nous ignorons également la nature des facteurs féminisants f d'A. vulgare et les 2 hypothèses proposées chez la drosophile peuvent être envisagées.

Il est à remarquer que l'action différentielle de la température élevée, selon qu'il s'agit du déterminant sexuel porté par l'hétérochromosome $\mathrm{W}$ d'une $q$ génétique - sur lequel la température n'agit pas - ou des facteurs féminisants $f$ - sur lesquels la température agit - suggère que ces facteurs $f$ sont d'une nature différente de celle des gènes sexuels de l'oniscoïde. 
Des données expérimentales récemment publiées (Legrand \& JuchaulT, 1984) et qui sont actuellement reprises sur une plus grande échelle - nous conduisent à penser que les facteurs $f$ auraient une origine épigénétique et seraient issus de la bactérie féminisante $F$. Ces données se rapportent, d'une part, à un passage de la thélygénie $T_{F}$ à l'arrhénogénie $A_{r}$ dans la descendance d'une femelle amphogène transformée en thélygène par inoculation de la bactérie $\mathrm{F}$, d'autre part, à l'existence de femelles masculinisables (donc privées de $F$ ) dans la descendance d'une femelle $T_{F}$. A notre connaissance, le seul exemple susceptible de correspondre également à l'intégration de facteurs héréditaires d'un Procaryote chez un Eucaryote concerne le règne végétal (revue in Gautheret, 1959). Néanmoins l'intervention de plasmides a été proposée pour expliquer les phénomènes de stérilité mâle chez les végétaux (revue in Pearson, 1981). Ce passage de $\mathrm{F}$ à $\mathrm{f}$, sous une forme inconnue intégrée - ou non aux chromosomes, s'effectuerait avec perte d'une partie de l'information génétique contenue dans l'ADN bactérien. En effet, les néo-femelles possédant le facteur f peuvent, comme les femelles génétiques, être masculinisées par l'hormone androgène, contrairement aux femelles hébergeant la bactérie $F$, qui sont réfractaires à cette hormone (Juchault \& Legrand, 1985).

Signalons enfin que, sur un plan théorique, l'existence d'un tel passage de $\mathrm{F}$ à $\mathrm{f}$ semble représenter l'une des hypothèses les plus vraisemblables pour expliquer le maintien d'individus mâles au sein de populations où s'est implantée la bactérie $F$. Effectivement, dans ces populations, les mâles sont essentiellement apportés par les descendances arrhénogènes, or l'arrhénauxèse intervenant dans ces descendances entraîne une raréfaction des femelles susceptibles d'engendrer des mâles; elle doit donc être compensée par un autre type d'apport de ces femelles (Juchault et al. 1980b ; Juchault \& Legrand, 1981a, 1981b ; Legrand \& Juchault, 1984).

Reçu le 17 février 1986.

Accepté le 5 mai 1986.

\section{Références bibliographiques}

Anxolabehere D., Périquet G., 1983. Système P-M de dysgénésie des hybrides, polymorphisme génétique et évolution des populations de Drosophila melanogaster. Génét. Sél. Evol., 45 (1), 31-44.

Bregliano J.C., Picard G., Bucheton A., Pelisson A., Lavigne J.M., L'Héritier P., 1980. Hybrid dysgenesis in Drosophila melanogaster. Science, 207, 606-611.

Bull J.J., 1983. Evolution of sex determining mechanisms. 316 pp., Benjamin/Cumings Pub. Comp., Meulo Parck, California.

Gautheret R.J., 1959. La culture des tissus végétaux. Techniques et réalisations. 863 pp., Masson, Paris.

GinSBURGer-Vogel Th., 1975. Influence de la température sur la différenciation sexuelle dans la descendance de certaines femelles chez Orchestia gammarellus. Bull. Soc. Zool. Fr., 100 (suppl. 1), 49-60.

Ginsburger-Vogel Th., 1985. Le déterminisme du sexe chez les Amphipodes. Bull. Soc. Zool. Fr., 110, 49-61.

Ginsburger-Vogel Th., Magniette-Mergault F., 1981a. The effects of temperature on sexual differentiation in the temperature sensitive thelygenic-intersexual offspring of Orchestia gammarellus (Pallas) (Amphipoda, Crustacea). I. Effects of temperature on pubescent females. Int. J. of Inv. Reprod., 4, 39-50.

Ginsburger-Vogel Th., Magniette-Mergault F., 1981b. The effects of temperature on sexual differentiation in the temperature sensitive thelygenic-intersexual offspring of Orchestia gam- 
marellus (Pallas) (Amphipoda, Crustacea). II. Effects of temperature during embryonic and post-embryonic development. Int. J. of Inv. Reprod., 4, 51-65.

Juchault P., Legrand J.J., 1976a. Modification de la sex-ratio dans les croisements entre différentes populations du Crustacé Oniscoïde Armadillidium vulgare Latr. Notion de déterminisme polygénique et épigénétique du sexe. Arch. Zool. Exp. Gén., 117 (1), 81-94.

Juchault P., Legrand J.J., 1976b. Etude génétique de l'intersexualité des mâles à ouvertures génitales femelles chez l'Oniscoïde Armadillidium vulgare Latr. : interprétation et modalités de la transmission héréditaire. C.R. Soc. Biol., 170, 429-433.

Juchault P., Legrand J.J., 1981a. Contribution à l'étude qualitative et quantitative des facteurs contrôlant le sexe dans les populations du Crustacé Isopode terrestre Armadillidium vulgare Latreille II. Populations hébergeant le facteur féminisant F (Bactéroïde intracytoplasmique). Arch. Zool. Exp. Gén., 122, 65-74.

Juchault P., Legrand J.J., 1981b. Contribution à l'étude qualitative et quantitative des facteurs contrôlant le sexe dans les populations du Crustacé Isopode terrestre Armadillidium vulgare Latreille. III. Populations n'hébergeant pas le facteur féminisant F (Bactéroïde intracytoplasmique). Arch. Zool. Exp. Gén., 122, 117-131.

Juchault P., Legrand J.J., 1985. Contribution à l'étude du mécanisme de l'état réfractaire à l'hormone androgène chez les Armadillidium vulgare Latr. (Crustacé, isopode, oniscoïde) hébergeant une bactérie féminisante. Gen. Comp. Endocrinol., 60, 463-467.

Juchault P., Martin G., Legrand J.J., 1980a. Induction par la température d'une physiologie mâle chez les néo-femelles et les intersexués du Crustacé Oniscoïde Armadillidium vulgare Latr., hébergeant un bactéroïde à action féminisante. Int. J. Inv. Reprod., 2, 223-235.

Juchault P., Legrand J.J., MocQuard J.P., 1980b. Contribution à l'étude qualitative et quantitative des facteurs contrôlant le sexe dans les populations du Crustacé Isopode terrestre Armadillidium vulgare Latreille. I. La population de Niort (Deux-Sèvres). Arch. Zool. Exp. Gén., 121, 3-27.

Legrand J.J., Juchault P., 1969. Le déterminisme de la monogénie chez les Oniscoïdes. C.R. Acad. Sci., Fr., 268, 1774-1777.

Legrand J.J., Juchault P., 1970. Modification expérimentale de la proportion des sexes chez les Crustacés Isopodes terrestres : induction de la thélygénie chez Armadillidium vulgare Latr. C.R. Acad. Sci., 270, 706-708.

Legrand J.J., Juchault P., 1972. Mise en évidence dans une population d'Armadillidium vulgare Latr. (Crustacé Isopode Oniscoïde) de deux types de lignées arrhénogènes en relation avec des facteurs épigénétiques à effet respectivement masculinisant et féminisant. C.R. Acad. Sc., Fr., 274, 1554-1557.

Legrand J.J., Juchault P., 1984. Nouvelles données sur le déterminisme génétique et épigénétique de la monogénie chez le Crustacé isopode terrestre Armadillidium vulgare Latr. Génét. Sél. Evol., 16 (1), 57-84.

L'Héritier Ph., 1962. Les relations du virus héréditaire de la Drosophile avec son hôte. Ann. Inst. Pasteur, 102, 511-526.

MARTIN G., 1981. Contribution à l'étude cytologique et fonctionnelle des systèmes de neurosécrétion des Crustacés Isopodes. Thèse Doctorat d'Etat, Université de Poitiers, $\mathbf{n}^{\circ} 331$.

Martin G., Juchault P., Legrand J.J., 1973. Mise en évidence d'un micro-organisme intracytoplasmique symbiote de l'Oniscoïde Armadillidium vulgare Latr., dont la présence accompagne l'intersexualité ou la féminisation totale des mâles génétiques de la lignée thélygène. C.R. Acad. Sc., Fr., 276, 2313-2316.

Minamori S., 1969. Extrachromosomal element delta in Drosophila melanogaster. I. Gene dependance of killing action and of multiplication. Genetics, 62, 583-596.

Mocquard J.P., Pavese A., Juchault P., 1980. Déterminisme de la reproduction saisonnière des femelles d'Armadillidium vulgare Latr. (Crustacé, Isopode, Oniscoïde). I. Action de la température et de la photopériode. Ann. Sc. Nat. Zool., Paris, 2, 91-97.

Ohanessian-Guillemain A., 1963. Etude de facteurs génétiques contrôlant les relations du virus o et de la drosophile son hôte. Ann. Génét., 5, 1-64.

Pearson O.H., 1981. Nature and mechanisms of cytoplasmic male sterility in plants : a review. Hortscience, 16, 482-487.

VANDel A., 1941. Recherches sur la génétique et la sexualité des Isopodes terrestres. VI. Les phénomènes de monogénie chez les Oniscoïdes. Bull. Biol. Fr. Belg., 75, 3, 316-363. 